\title{
Quality Assurance for Clinical fMRI
}

The functional MRI (fMRI) procedure has several sources of variance that determine the success of the examination. These include the scanner, patient, and paradigm. A full discussion of these items has been given elsewhere (Thulborn, 1999; Thulborn and Gisbert, 2000). As blood oxygenation level dependent (BOLD) contrast is a small effect, high signal-to-noise performance is mandatory. Because the preparation of a functional activation map requires averaging multiple images over time, the scanner must produce high temporal stability of the signal intensity. There are many determinants of such performance but not all possibilities need to be checked separately. An adequate approach has been to verify total system performance under the conditions of a functional MRI study on a phantom. This testing is done daily prior to patient studies.

\section{SCANNER STABILITY}

BASIC

The scanner must be able to produce many high-quality, temporally-stable images continuously over many minutes. Daily assessment of stability and image quality can ensure that fMRI studies are acquired under optimal conditions.

Table A6.2.1 lists the hardware requirements for the BOLD contrast fMRI component of a comprehensive MRI examination. The radiofrequency coil should be a high performance volume coil for whole brain imaging. The gradient set should be capable of single-shot, echo-planar imaging. The typical gradient strength for high quality echo-planar imaging is on the order of 25 to $40 \mathrm{mT} / \mathrm{m}$ with slew rates of 75 to $150 \mathrm{mT} / \mathrm{m} / \mathrm{sec}$ or better.

\section{Materials}

Spherical phantom in loader with doped water, 16-cm diameter

\section{Equipment setup}

1. Place the phantom on the scanner table. Adjust the table such that the laser light is at the center of the phantom. Turn off the laser light and move the phantom to the center of the magnet.

\section{Quality assurance sequence}

2. Perform scans using the protocol listed in Table A6.2.2.

Table A6.2.1 Equipment for fMRI

\begin{tabular}{ll}
\hline Magnet & $1.5 \mathrm{~T}$ or greater \\
Coil type & Volume transmit/receive head coil \\
Gradient Set & $25-40 \mathrm{mT} / \mathrm{m}, 75-150 \mathrm{mT} / \mathrm{m} / \mathrm{sec}$ \\
Motion cushions & Useful \\
Peripheral gating & Unnecessary except for monitoring \\
Cardiac gating & Unnecessary \\
Respiratory gating & Unnecessary \\
Respirator & Contraindication \\
Oxygen & No \\
Use of contrast agents & No \\
\hline
\end{tabular}

Contributed by Keith R. Thulborn and Denise Davis

Current Protocols in Magnetic Resonance Imaging (2001) A6.2.1-A6.2.4

Copyright $(\subseteq 2001$ by John Wiley \& Sons, Inc. 
Table A6.2.2 Stability of MR Signal Intensity for Echo-Planar Imaging at 1.5 and $3.0 \mathrm{~T}$

\begin{tabular}{ll}
\hline Patient position & NA \\
Scan type & Gradient echo echo-planar \\
Imaging plane (orientation) & Transverse \\
Central slice or volume center & Center \\
Echo time $\left(T_{\mathrm{E}}\right)$ & $50 \mathrm{msec}$ at $1.5 \mathrm{~T}, 25 \mathrm{msec}$ at $3.0 \mathrm{~T}$ \\
Repeat time $\left(T_{\mathrm{R}}\right)$ & $2000 \mathrm{msec}$ \\
Flip angle $(\mathrm{FA})$ & $90^{\circ}$ \\
Fields of view $\left(\mathrm{FOV}, \mathrm{x}, \mathrm{FOV}_{\mathrm{y}}\right)$ & $200 \mathrm{~mm}, 200 \mathrm{~mm}$ \\
Resolution $(\Delta x, \Delta y)$ & $3.1 \mathrm{~mm}, 3.1 \mathrm{~mm}$ \\
Number of data points collected $\left(N_{\mathrm{x}}, N_{\mathrm{y}}\right)$ & $64,64(\mathrm{ramp}$ sampling $)$ \\
Display matrix $\left(D_{\mathrm{x}}, D_{\mathrm{y}}\right)$ & 64,64 \\
Slice thickness $(\Delta z)$ & $5 \mathrm{~mm}$ at $1.5 \mathrm{~T}, 3 \mathrm{~mm}$ at $3 \mathrm{~T}$ \\
Number of slices & $10(521 \mathrm{images}$ total $)$ \\
Slice gap & $12.5 \mathrm{~mm}$ \\
Number of acquisitions $\left(N_{\mathrm{acq}}\right)$ & 1 \\
Swap read and phase encoding & No \\
Slice locations & Graphic prescription, 10 slices \\
Saturation pulses & None \\
Ramp sampling & On \\
Scan time & $17 \mathrm{~min}, 12$ sec \\
\hline
\end{tabular}

The limit of 512 images is a current limit on file data length on one scanner for a standard echo-planar sequence and represents a convenient maximum acquisition length. This study gives day to day measures of signal stability, signal-to-noise performance, and ghosting levels.

3. Compare these measurements with baseline values obtained at the time of acceptance from the manufacturer. Deviations from baseline indicate the need for a service call.

The reliability of fMRI results from a scanner showing fluctuations in these quality assurance parameters cannot be assured.

\section{COMMENTARY}

\section{Background Information}

There are many factors that determine overall scanner performance. It is important to ensure that the initial installation has been done correctly and that manufacturer specifications have been met. A complete report on the installation should be forthcoming from the vendor. This report will include magnet homogeneity specified in Hertz over different diameters of a spherical volume. It will include radio frequency (RF) testing of the room at all potential problem sources including the door, window, and penetration panel. These measurements should typically be better than $100 \mathrm{~dB}$ at the operating frequency of the scanner. Signal-tonoise ratio, ghost-to-signal measurement, and a stability performance, such as with this protocol, should be established close to acceptance of the scanner so that a performance history can be maintained. A scanner installed in an ill-prepared site can be severely compromised. Having accepted a well installed scanner, performance may degrade slowly over time. This can happen for many reasons and it is important to separate scanner from sitting issues.

\section{Critical Parameters and Troubleshooting}

Many sources of potential problems reflect the site rather than the scanner. This can be suggested as noise floor increases without changes in signal intensity. The RF seals on the door and penetration panel are likely sources. Sometimes electrical circuits within the magnet room can become noise sources. Care should be taken to never breach the wave guides with conducting materials. Wire used to provide electrical connections through the wave guides

A6.2.2 


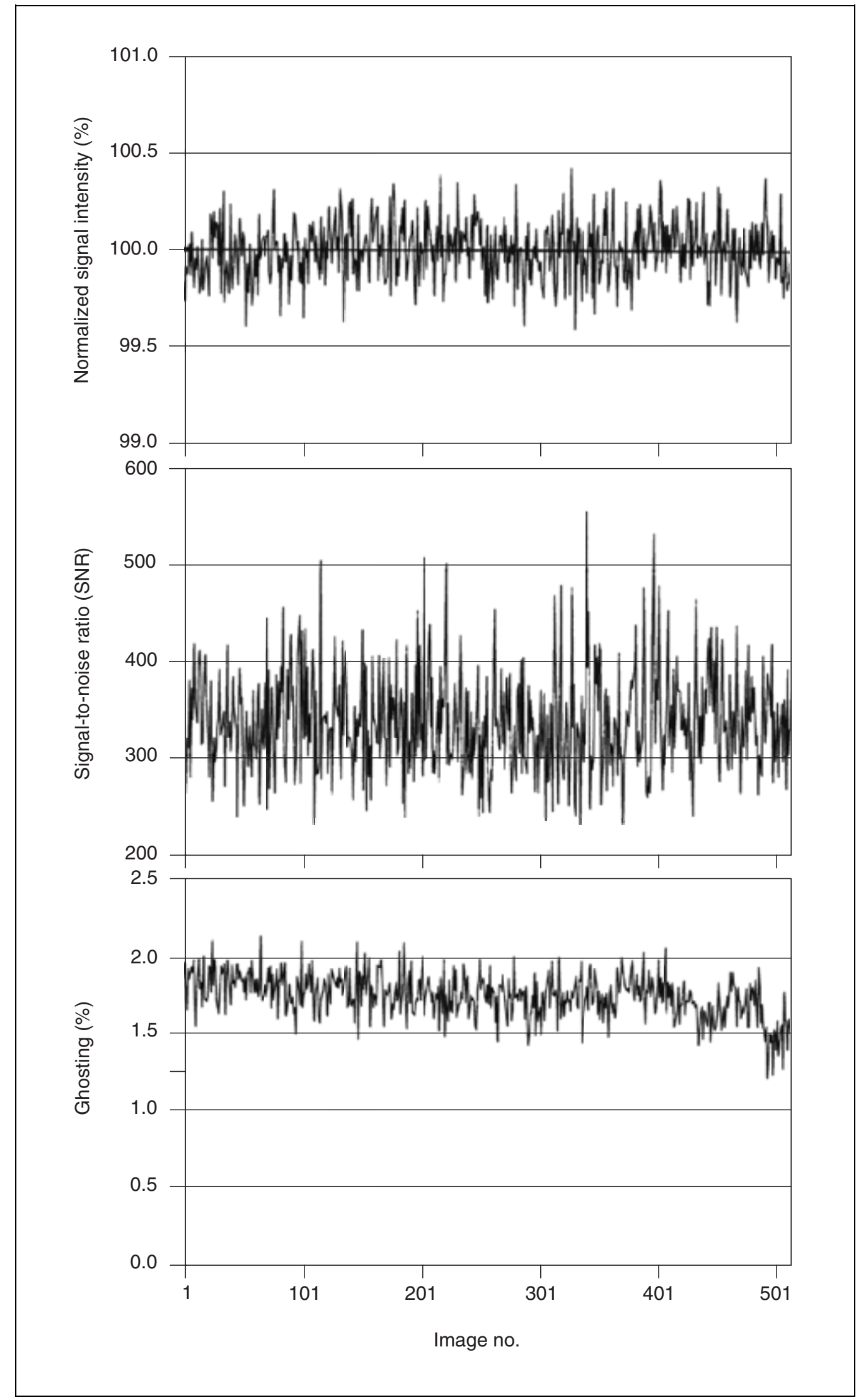

Figure A6.2.1 Representative results of the quality assurance protocol for one day from a $1.5 \mathrm{~T}$ scanner. (A) Normalized signal intensity over 512 images showing a peak-to-peak variation of less than 1\%. (B) Signal-to-noise ratio over 512 images showing a mean value above 300 . (C) Percentage ghosting over 512 images showing a mean value of less than $2 \%$. 
for accessory equipment used by some investigators in fMRI experiments are a notorious source of this problem.

RF doors have variable quality and longevity. Some pneumatically sealed doors have been shown to maintain excellent performance for years while others have degraded in months. Careful maintenance of the site is a necessary element of quality assurance.

The scanner is not without its own issues. An increasing power requirement for determining the $90^{\circ}$ nutation (flip) angle for the head RF coil suggests a coil problem. A decreased signal without a change in the noise figure or power requirement may point to a preamplifier problem. Low signal also comes from poor magnet homogeneity which can be caused by a simple paper clip inadvertently flying into the magnet. Many of the details of troubleshooting are best left to the field service engineer; however, someone has to be aware of the problem in order to contact service, hopefully before patient care is compromised. Daily quality assurance ensures timely response to potential problems.

\section{Anticipated Results}

Typical results are shown in Figure A6.2.1. Typically, the peak-to-peak signal variation should be less than $1 \%$ over the 17 min acquisition. Although not an absolute value, the signal-to-noise ratio for this scanner and method of measurement is over 330. Similarly, the ghosting level, reflecting intrinsic gradient performance and eddy current correction, is under $2 \%$. These parameters are useful for overall assessment of the scanner electronics, gradient performance, RF noise isolation of the scanner room, and magnet homogeneity.

\section{Literature Cited}

Thulborn, K.R. 1999. Quality assurance in clinical and research echo-planar functional MRI. In Medical Radiology: Diagnostic Imaging and Radiation Oncology. Functional MRI (C. Moonen and P. Bandettini, eds.) pp. 337-345. SpringerVerlag, Berlin.

Thulborn, K.R. and Gisbert, A. 2000. Clinical Applications of Mapping Neurocognitive Processes in the Human Brain with Functional MRI. (P. Matthews and P. Jezzard, eds.) Oxford University Press, Oxford. In press.

Contributed by Keith R. Thulborn and Denise Davis

University of Illinois at Chicago

Chicago, Illinois 